Preface

\title{
Hot Topics V
}

\author{
Emmanuel J. Favaloro, PhD, FFSc (RCPA) ${ }^{1}$ \\ ${ }^{1}$ Department of Haematology, Institute of Clinical Pathology and \\ Medical Research (ICPMR), Westmead Hospital, Westmead, Australia
}

Semin Thromb Hemost 2014;40:5-10.

Welcome to the first issue of Seminars in Thrombosis and Hemostasis for 2014, and the fifth issue compilation entitled "Hot Topics."1-4 The previous four "Hot Topics" issues have been very popular with our readership, as identified in subsequent analyses. ${ }^{5-9}$ Although Seminars in Thrombosis $\mathcal{E}$ Hemostasis is primarily a theme-driven publication, the occasional opportunity arises to publish composite issues containing more wide-ranging articles of current interest and controversy. This opportunity presents itself once again as a result of the most recent (2013) Eberhard F. Mammen Young Investigator awards. ${ }^{9}$ Accordingly, I am very pleased to present the latest "Hot Topics" issue (Part V), including articles from our six most recent Eberhard F. Mammen Young Award winners. ${ }^{9}$ The remaining articles from this issue derive from various contributors, including several from editorial members of this journal.

The issue begins with several articles that concern the hemostasis triad of ADAMTS-13 (a disintegrin and metalloprotease with thrombospondin type I repeats-13), von Willebrand factor (VWF), von Willebrand disease (VWD), and/or various interrelationships. The first article is from one of our recent Young Investigator awardees, Andrew Yee, ${ }^{9}$ and his colleague, Colin Kretz, who describe the relationship between the structure of VWF and its various hemostatic functions. ${ }^{10}$ VWF comprises many subunits, which themselves are composed of many repeated domains/assemblies possessing characteristic function(s). Organization of these domains/assemblies into a multimeric structure effectively links and replicates these functions. Whereas low-molecularweight forms of VWF have diminished hemostatic potential, ultralarge VWF (ULVWF) is potentially thrombogenic when in excess, as occurs for example in TTP (thrombotic thrombocytopenic purpura). Each domain/assembly within VWF influences the synthesis, assembly, secretion, or hemostatic potential of plasma VWF. Hemodynamic forces also regulate the conformation of the $\mathrm{A} 2$ domain and thus, its accessibility to proteolytic enzyme(s) that regulate VWF's hemostatic potential, including ADAMTS-13. Distinct binding to the

Address for correspondence Emmanuel J. Favaloro, PhD, FFSc (RCPA), Department of Haematology, Institute of Clinical Pathology and Medical Research (ICPMR), Westmead Hospital, Westmead, Australia (e-mail: emmanuel. favaloro@health.nsw.gov.au).
VWF D'D3 assemblies stabilizes coagulation factor VIII, whereas the VWF A1 and A3 domains facilitate platelet capture onto exposed collagen(s) at sites of vascular injury. In summary, a fuller understanding of VWF, and its structure and form, may in the future potentially guide novel therapeutics for clotting or bleeding disorders.

The second article, from Eerenberg and Levi, extends this discussion to the potential therapeutic benefit of targeting ADAMTS- 13 activity. ${ }^{11}$ As partially explored by Yee and Kretz in the first article, ${ }^{10}$ ADAMTS- 13 is a protease capable of cleavage of UL VWF, thereby decreasing its prothrombotic properties. There are a myriad of pathological clinical conditions associated with decreased levels of ADAMTS-13, often co-associated with thrombotic microangiopathy, and including TTP. Nevertheless, other diseases also exist in which ADAMTS-13 levels are decreased and thus where reduced cleavage of VWF may play a pathophysiological role. Hence, targeting of VWF cleavage by pharmacological modulation of ADAMTS-13 level and activity is a feasible approach to treating some of these conditions. Eerenberg and Levi thus review the available evidence for a role of ADAMTS- 13 in various disease states and the potential therapeutic benefit of restoration of ADAMTS-13 activity in these conditions.

The third article in this issue is from another of our recent Young Investigator awardees, ${ }^{9}$ Kent Chapman, and his colleague, Sam Yuen, ${ }^{12}$ with continued discussion on TTP and ADAMTS-13, focusing on past, present, and potential future therapy for TTP. While clinical recognition of this disease has been evident for almost 90 years, its pathological basis has only relatively recently been elucidated. Consequently, options for treating TTP have evolved only slowly and currently involve intensive plasma exchange, often with immune modulation. Although this treatment has seen dramatic improvement in patient survival rates, current understanding of TTP may witness emergence of new treatments that specifically target the root cause of TTP. Some of these targeted approaches may even see the beginning of plasma free treatments for TTP, with potentially faster recoveries and fewer long-term side effects.
Issue Theme Hot Topics V; Guest Editor, Emmanuel J. Favaloro, PhD, FFSC (RCPA).
Copyright $\odot 2014$ by Thieme Medical Publishers, Inc., 333 Seventh Avenue, New York, NY 10001, USA. Tel: +1(212) 584-4662.
DOI http://dx.doi.org/ 10.1055/s-0034-1364321. ISSN 0094-6176. 
The fourth article, by Veronica Flood, another Eberhard F. Mammen Young Investigator award winner, ${ }^{9}$ turns our attention from thrombotic conditions associated with excess UL VWF to the bleeding-associated condition VWD, which essentially arises from defects and/or deficiencies in VWF. VWD is considered the most common bleeding disorder worldwide, ${ }^{14}$ and its diagnosis merits consideration of personal and family history of bleeding symptoms along with confirmatory laboratory testing. Because laboratory testing yields quantifiable results, a diagnosis of VWD based on overreliance of test results may occur. However, these tests have several potential sources of error, with both intrinsic and extrinsic factors affecting all assays and potentially contributing to either falsely normal or falsely abnormal test results. Flood discusses presently available assays as well as new developments in diagnostic testing and concludes that a clear understanding of the limitations of VWD testing is helpful for ensuring correct diagnosis of affected patients.

The fifth article in this issue, by Dentali and colleagues, ${ }^{15}$ also in part continues some discussion of VWF, but only briefly in the context of $A B O$ blood group influence on hemostasis. The relationship between $O$ blood type and bleeding risk, and between non-O blood type and the risk of venous thromboembolism and coronary artery disease, has been previously explored by members of this group in this journal and elsewhere. ${ }^{16-19}$ Nevertheless, the association of the $\mathrm{ABO}$ blood type with arterial thrombotic events (i.e., myocardial infarction and ischemic stroke) is less well characterized, and accordingly, Dentali and colleagues ${ }^{15}$ have conducted a systematic review and meta-analysis of the existing literature. They interestingly report that compared with control groups, the prevalence of non-O blood group is significantly higher in patients with myocardial infarction and ischemic stroke.

The sixth article in this issue, by Lippi et al, continues the discussion of cardiovascular risk, this time in relation to Ecigarettes. $^{20}$ Giuseppe Lippi is a regular contributor to Seminars in Thrombosis \& Hemostasis, and a previous winner of one of the Eberhard F. Mammen most popular awards. ${ }^{8}$ In this latest contribution, Lippi and colleagues first set the scene, reminding readers that cigarette smoking is the most important cause of premature death, and currently listed as a major independent risk factor for cardiovascular disease. Because of current restrictive measures and widespread control policies by governments and health authorities, tobacco companies have initiated aggressive marketing strategies in favor of smokeless tobacco, including electronic nicotine delivery systems, also known as electronic cigarettes or e-cigarettes. Although the regular use of these devices appears less hazardous than traditional cigarettes or other forms of smokeless tobacco, recent studies have shown that a variety of potentially harmful substances, especially nicotine, ultraparticles, and volatile organic compounds, may be effectively inhaled or liberated in exhaled air during repeated e-cigarette puffing. This would enhance the risk of cardiac arrhythmias and hypertension, which may predispose some users to increased risk of cardiovascular events, which may be further magnified by other potential side effects such as arrhythmias, increased respiratory and flow respiratory resistance. Cases of intoxication have also been described, as large amounts of nicotine and other harmful compounds may be effectively absorbed. The authors conclude that because the use of e-cigarettes is continuously rising, and considered a potentially effective method for smoking cessation, more focused research is urgently needed to definitely establish the cardiovascular safety of these devices.

The seventh article in this issue is by another of our Young Investigator Awardees, Sarah O'Brien, ${ }^{9}$ who reports on contraception-related venous thromboembolism in adolescents. $^{21}$ Venous thromboembolism (VTE) is a rare but serious complication of combined hormonal contraception. While the absolute risk of VTE is low in adolescents, thrombotic events in contraception users younger than 20 years of age account for 5 to $10 \%$ of total contraception-related VTE events in population studies, due to the high frequency of contraception use in adolescents. An increased risk of VTE exists not only with oral contraceptives, but also the contraceptive patch and vaginal ring. Most adolescents who experience contraception-related VTE have additional transient or inherited thrombotic risk factors at the time of VTE. In a loose connection with the previous article by Lippi et al, ${ }^{20}$ one potential risk factor in this scenario is cigarette smoking. ${ }^{21}$ While the presence of inherited thrombophilia impacts the risk of contraception-related VTE, O'Brien advises that thrombophilia screening before contraception prescribing should be targeted only to high-risk populations. The author concludes that pediatric institutions, caregivers, and young women need to be aware of the risk of VTE with estrogencontaining contraception and maintain a high index of suspicion for this complication in women using these agents.

Discussion on thrombosis continues in the eighth article in this issue, also by another of our Young Investigator Awardees, Vivian $\mathrm{Du},{ }^{9}$ who with the assistance of several colleagues, provides several new insights into the role of erythrocytes in thrombus formation. ${ }^{22}$ For quite a long time, the role of erythrocytes in thrombus formation has been regarded as passive, simply by their influence on rheology. Erythrocytes are known, due to their abundance and size, to push platelets to the vascular wall (laminar shearing). This results in an increased platelet delivery at the vascular wall enabling platelets to seal off vascular damage preventing excessive blood loss. Recently, however, new evidence is emerging for more active roles for erythrocytes in influencing thrombus formation. For example, erythrocytes are the main suppliers of phosphatidylserine-exposing membranes as used in coagulation and resulting in fibrin formation. In addition, the authors' group has found that the ICAM- $4-\alpha_{\mathrm{II}} \beta_{3}$ interaction mediates erythrocyte-platelet interaction in flowing blood. By inhibiting this interaction thrombin formation is decreased, as is the incorporation of erythrocytes into a thrombus. In total, this review provides in-detail information on existing and new proposed mechanisms whereby erythrocytes may be involved in thrombus formation.

Also continuing the discussion on thrombosis is Girolami and colleagues, ${ }^{23}$ who in the ninth article of this issue explore the diagnostic challenge provided by venous thrombosis in 
unusual anatomical sites. ${ }^{23}$ Of potential interest to readers is the fact that Drs Bruno and Antonio Girolami, two of the authors of the current article, were winners of one of the inaugural Eberhard Mammen Most Popular article awards. ${ }^{5}$ Venous thrombosis usually involves the veins of the limbs, most frequently the leg veins. However, all other venous districts may on occasion be affected by the thrombotic process. Moreover, although the thrombotic occlusion of the veins of a given region may show typical signs and symptoms, in other cases, the picture may not be clear and a high degree of clinical suspicion will be required to ensure the correct approach to patient diagnosis and management. Thrombosis of retinal and jugular veins, right heart thrombosis including thrombosis of coronary sinus and thrombosis of the azygos system may be included in this group. In addition, thromboses of umbilical, renal, ovarian, spermatic, and iliac veins also require attention. Finally, the dorsal veins of the penis may also be affected by thrombotic events. The main clinical features of these thromboses are reviewed in the current article, ${ }^{23}$ inclusive of suggestions for a correct diagnostic approach; the importance of sonography and of other imaging techniques is also emphasized. The authors conclude that prompt diagnosis of these thromboses is of paramount importance since most still cause severe systemic complications (pulmonary embolism, sepsis, heart failure).

The 10th article of this issue, by Carubbi and colleagues, ${ }^{24}$ deals with cytofluorimetric platelet analysis. The authors begin by reminding us that blood platelets are highly specialized cells that drive hemostatic events and tissue repair mechanisms at the site of vascular injury, and then advising that their peculiar morphology and certain functional characteristics can be analyzed by flow cytometry (FCM). Specifically, platelet activation, a hallmark of prothrombotic states and inflammatory conditions, is associated with changes in expression of both surface and intracellular antigens that are recognized by specific monoclonal antibodies. Assessment of platelet activation status as ex vivo or in vitro reactivity to specific agonists has also become relevant in particular conditions (including, cardiovascular diseases, hematologic malignancies, and monitoring of pharmacological anti-aggregation). Aberrant surface marker expression that characterizes inherited and acquired platelet function disorders can also be detected by FCM. In total, this review provides a rich overview of the main applications of FCM in platelet analyses, which are relevant for both research and clinical settings.

The remaining articles in this issue of Seminars in Thrombosis $\mathcal{E}$ Hemostasis largely relate to therapy in thrombosis and/or associated problems. The first in this series of articles is from Ageno and colleagues on behalf of the IRSVT study group, ${ }^{25}$ describing current treatment strategies and factors associated with therapeutic decisions in a large prospective cohort of unselected splanchnic vein thrombosis (SVT) patients. Treatment of SVT is clinically challenging due to heterogeneity of clinical presentations, increased bleeding risk and lack of evidences from clinical trials. The authors therefore performed an international registry that enrolled a total of 613 patients, the majority of whom had portal vein thrombosis. Most common risk factors included cirrhosis (27.8\%), solid cancer (22.3\%), and intra-abdominal inflammation/infection (11.7\%); in $27.4 \%$ of patients, SVT was idiopathic. During the acute phase, 470 (76.7\%) patients received anticoagulant drugs, whereas 136 patients (22.2\%) remained untreated. Incidental diagnosis, single vein thrombosis, gastrointestinal bleeding, thrombocytopenia, cancer, and cirrhosis were significantly associated with no anticoagulant treatment. The decision to start patients on vitamin $\mathrm{K}$ antagonists (VKAs) after an initial course of parenteral anticoagulation was significantly associated with younger age, symptomatic onset, multiple veins involvement, and unprovoked thrombosis. Although a nonnegligible proportion of SVT patients did not receive anticoagulant treatment, the majority received the same therapies recommended for patients with usual sites thrombosis, with some differences driven by the site of thrombosis and the pathogenesis of the disease.

The next article ${ }^{26}$ is by Cuker, one of our previous Eberhard F. Mammen Young Investigator Awardees, ${ }^{7}$ and concerns the clinically significant pathologic disorder heparin-induced thrombocytopenia (HIT), which predisposes affected individuals to thrombosis. Diagnosis of HIT relies on a compatible clinical picture and laboratory evidence of antiplatelet factor 4 (PF4)/heparin antibodies that activate platelets in a heparindependent manner. Laboratory tests include immunoassays such as the PF4/heparin enzyme-linked immunosorbent assay (ELISA) and functional tests such as the ${ }^{14} \mathrm{C}$-serotonin release assay and heparin-induced platelet activation assay. Although rapid and accurate diagnosis is paramount to avoid the perils of misdiagnosis, the available tests are insufficiently sensitive and specific on their own to adequately diagnose or exclude HIT. Clinical evaluation is therefore critical, and may be guided by scoring systems such as the 4Ts and HIT Expert Probability (HEP) score. Clinical scoring systems and commercially available immunoassays in essence have high sensitivity, but modest specificity. Functional assays are more specific, but are technically demanding. Novel laboratory assays with faster turnaround times, greater specificity, and lesser technical complexity are in development. A Bayesian approach that combines the 4T score and the PF4/heparin ELISA result may be useful to estimate the probability of HIT and guide clinical decision-making.

The next article, by the last of our current Eberhard F. Mammen Young Investigator Award winners, Julie Tange, ${ }^{27}$ and colleagues from the Mayo Clinic, describes their approach to the local verification and assignment of geometric mean normal prothrombin time (MNPT) and international sensitivity index (ISI) values across various instruments in a large network of laboratories. VKA therapy using drugs such as warfarin currently remains the most common therapy for treatment and prevention of thrombosis recurrence. However, dosing with VKAs relies on accurate measurements of the international normalized ratio (INR), which in turn is calculated from the patient's prothrombin time (PT), divided by the MNPT, and then brought to the power of the ISI of the thromboplastin used. However, ISI assignments of certain reagent/instrument combinations are frequently unavailable, especially when the reagent and instrument are not from the 
same manufacturer. ${ }^{28}$ The effort to be in compliance with widely endorsed international expert guidelines by locally verifying or assigning an ISI to an unsupported reagent/instrument combination is further hindered by a lack of USA Food and Drug Administration (FDA)-approved certified plasmas designated for a particular reagent/instrument combination. ${ }^{28}$ The Mayo group therefore aimed to develop a process to verify/ assign ISI and MNPT to a single thromboplastin reagent from one manufacturer across multiple instruments including several from another manufacturer and across several campuses of their institution. The process they describe provided a reproducible approach to assigning ISIs on unsupported reagent/instrument combinations, and further confirmed that ISIs of the same PT reagent differ significantly on different instruments. Their findings also substantiate the requirement for evaluations and validation of ISIs for different reagent/ instrument combinations.

The final two articles in this issue largely relate to the new direct oral anticoagulants (DOACs), the subject of many recent previous articles in this journal and elsewhere. ${ }^{29-37}$ The first by Zolfaghari et al describes the development of a tool to identify patient's preference for these direct drugs or alternatively for VKA therapy. ${ }^{38}$ DOACs, including dabigatran, rivaroxaban, and apixaban were developed for treatment of thromboembolic diseases to overcome limitations and to improve the efficacy/safety profile of VKAs. Although the preference of patients, for example, with atrial fibrillation, for long term, potentially life-long, prevention of future embolic events by therapy with VKA or DOAC is undisputedly important, the objective assessment of this preference is difficult due to social interactions and psychological factors. Based on results of structured interviews, followed by several pilot studies on the preparedness to use DOACs as anticoagulant and on the motivation of patients to participate in clinical studies with DOACs, Zolfaghari et al have developed a tool for objective identification of patient's preference, important also for maximizing adherence to therapy, and to thereby potentially reduce undertreatment. ${ }^{38}$

The final article in this issue is by Harenberg and colleagues, and describes the determination of DOACs from human serum samples. ${ }^{39}$ The DOACs inhibit either thrombin or factor Xa and effectively preventing thromboembolic complications using fixed doses without need for laboratoryguided dose adjustment. However, occasional assessment of DOACs may be required for special patient populations such as those with acute deterioration of renal function due to any disease, before surgical interventions, during bleeding or thrombotic episodes while on therapy with DOACs, the elderly and youngest populations, unexpected pregnancy, suspicion of overdose and toxication, and to control adherence to therapy. ${ }^{32-37}$ Current tests assessed to determine the concentration or activity of DOACs have all utilized plasma samples, except for a single previous study ${ }^{31}$ using urine samples. Nevertheless, serum samples have several advantages over plasma samples such as no need of blood sampling with a specific coagulation tube, reduced preanalytical errors associated with clotting tests, and longer sample storage stability. The authors therefore assessed the determination of these DOACs in serum samples of patients on treatment, and tests in general performed well compared with comparative results using plasma samples.

As always, I thank all the authors to this issue of Seminars in Thrombosis \& Hemostasis for their original and comprehensive contributions. However, I would especially thank the Eberhard F. Mammen Young Investigator Award winners for their submissions. I also hope that the readership of this journal finds the entire issue of considerable interest. This will be determined in time, as measured and established for previous issues of "Hot Topic" compilations. ${ }^{1-9}$

\section{References}

1 Favaloro EJ. Hot topics I: a potpourri of current issues and controversies in thrombosis and hemostasis. Semin Thromb Hemost 2007;33(8):723-726

2 Favaloro EJ. Hot topics II: an editorial collection of current issues and controversies in thrombosis and hemostasis. Semin Thromb Hemost 2008;34(1):3-6

3 Favaloro EJ. Hot topics III. Preface. Semin Thromb Hemost 2012; 38(1):1-4

4 Favaloro EJ. Hot Topics IV. Semin Thromb Hemost 2013;39(1):1-4

5 Favaloro EJ. Editorial. Winners of the Inaugural Eberhard F. Mammen Award for Most Popular Article. Semin Thromb Hemost 2009; 35:587-590

6 Favaloro EJ. Winners of the 2010 Eberhard F. Mammen award for most popular article during 2008-2009. Semin Thromb Hemost 2010;36(7):685-692

7 Favaloro EJ. 2011 Eberhard F. Mammen award announcements. Semin Thromb Hemost 2011;37(5):431-439

8 Favaloro EJ. 2012 Eberhard F. Mammen award announcements. Semin Thromb Hemost 2012;38(5):425-432

9 Favaloro EJ. 2013 Eberhard F. Mammen award announcements. Semin Thromb Hemost 2013;39(6):567-574

10 Yee A, Kretz CA. von Willebrand factor: form for function. Semin Thromb Hemost 2014;40(1):17-27

11 Eerenberg ES, Levi M. The potential therapeutic benefit of targeting ADAMTS13 activity. Semin Thromb Hemost 2014;40(1): 28-33

12 Chapman K, Yuen S. Therapy for thrombotic thrombocytopenia purpura: past, present, and future. Semin Thromb Hemost 2014; 40(1):34-40

13 Flood VH. Perils, problems, and progress in laboratory diagnosis of von Willebrand disease. Semin Thromb Hemost 2014;40(1):41-48

14 Favaloro EJ. Von Willebrand disease: local diagnosis and management of a globally distributed bleeding disorder. Semin Thromb Hemost 2011;37(5):440-455

15 Dentali F, Sironi AP, Ageno W, Crestani S, Franchini M. ABO blood group and vascular disease: an update. Semin Thromb Hemost 2014;40(1):49-59

16 Dentali F, Sironi AP, Ageno W, et al. Relationship between ABO blood group and hemorrhage: a systematic literature review and meta-analysis. Semin Thromb Hemost 2013;39(1):72-82

17 Franchini M, Liumbruno GM. ABO blood group: old dogma, new perspectives. Clin Chem Lab Med 2013;51(8):1545-1553

18 Franchini M, Rossi C, Mengoli C, et al. ABO blood group and risk of coronary artery disease. J Thromb Thrombolysis 2013;36(3): 286-287

19 Franchini M, Favaloro EJ, Targher G, Lippi G. ABO blood group, hypercoagulability, and cardiovascular and cancer risk. Crit Rev Clin Lab Sci 2012;49(4):137-149

20 Lippi G, Favaloro EJ, Meschi T, Mattiuzzi C, Borghi L, Cervellin G. Ecigarette and cardiovascular risk: beyond science and mysticism. Semin Thromb Hemost 2014;40(1):60-65 
21 O'Brien SH. Contraception-related venous thromboembolism in adolescents. Semin Thromb Hemost 2014;40(1):66-71

22 Du VX, Huskens D, Maas C, Al Dieri R, de Groot PG, de Laat B. New insights into the role of erythrocytes in thrombus formation. Semin Thromb Hemost 2014;40(1):72-80

23 Girolami A, Treleani M, Bonamigo E, Tasinato V, Girolami B. Venous thrombosis in rare or unusual sites: a diagnostic challenge. Semin Thromb Hemost 2014;40(1):81-87

24 Carubbi C, Masselli E, Gesi M, et al. Cytofluorimetric platelet analysis. Semin Thromb Hemost 2014;40(1):88-98

25 Ageno W, Riva N, Schulman S, et al; on behalf of the IRSVT study group. Antithrombotic treatment of splanchnic vein thrombosis: results of an International registry. Semin Thromb Hemost 2014; 40(1):99-105

26 Cuker A. Clinical and laboratory diagnosis of heparin-induced thrombocytopenia: an integrated approach. Semin Thromb Hemost 2014;40(1):106-114

27 Tange JI, Grill D, Koch CD, et al. Local verification and assignment of mean normal prothrombin time and international sensitivity index values across various instruments: recent experience and outcome from North America. Semin Thromb Hemost 2014;40(1):115-120

28 Favaloro EJ, Plebani M, Lippi G. Regulation in hemostasis and thrombosis: part I-in vitro diagnostics. Semin Thromb Hemost 2013;39(3):235-249

29 Harenberg J, Giese C, Marx S, Krämer R. Determination of dabigatran in human plasma samples. Semin Thromb Hemost 2012; 38(1):16-22

30 Harenberg J, Erdle S, Marx S, Krämer R. Determination of rivaroxaban in human plasma samples. Semin Thromb Hemost 2012; 38(2):178-184
31 Harenberg J, Du S, Krämer S, et al. Novel methods for assessing oral direct factor Xa and thrombin inhibitors: use of point-of-care testing and urine samples. Semin Thromb Hemost 2013;39(1): 66-71

32 Salmela B, Joutsi-Korhonen L, Armstrong E, Lassila R. Active online assessment of patients using new oral anticoagulants: bleeding risk, compliance, and coagulation analysis. Semin Thromb Hemost 2012;38(1):23-30

33 Schulman S, Majeed A. The oral thrombin inhibitor dabigatran: strengths and weaknesses. Semin Thromb Hemost 2012;38(1): 7-15

34 Rojas-Hernandez CM, Garcia DA. The novel oral anticoagulants. Semin Thromb Hemost 2013;39(2):117-126

35 Favaloro EJ, Bonar R, Butler J, Marsden K. Laboratory testing for the new oral anticoagulants: a review of current practice. Pathology 2013;45(4):435-437

36 Favaloro EJ, Lippi G. The new oral anticoagulants and the future of haemostasis laboratory testing. Biochem Med (Zagreb) 2012; 22(3):329-341

37 Di Minno A, Spadarella G, Prisco D, Franchini M, Lupoli R, Di Minno MN. Clinical judgment when using coagulation tests during direct oral anticoagulant treatment: a concise review. Semin Thromb Hemost 2013;39(7):840-846

38 Zolfaghari S, Harenberg J, Froelich L, Wehling M, Weiss C. Development of a tool to identify patients' preference for vitamin- $\mathrm{K}$ antagonist or direct oral anticoagulant therapy. Semin Thromb Hemost 2014;40(1):121-128

39 Harenberg J, Kraemer S, Du S, Giese C, Schulze A, Kraemer R, Weiss C. Determination of direct oral anticoagulants from human serum samples. Semin Thromb Hemost 2014;40(1):129-134 